\title{
Driving Technologies for the Design of Additive Manufacturing Systems
}

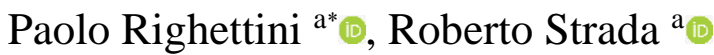 \\ ${ }^{a}$ Department of Engineering and Applied Sciences, University of Bergamo, 24044 Dalmine (BG), Italy.
}

\begin{abstract}
In recent years, the Additive Manufacturing (AM) technology, belonging to the most comprehensive Net Shape Forming family, has shown a growing trend due to the increasing quality of the built product. These results may open the application of the AM to the industrial field, moving the application from laboratories to the plant floor. This step requires machines capable of executing the technology process of AM with the requirements of the industrial environment, concerning, for example, production speed, reliability, robustness, and process stability. The design of such a type of machinery requires a systematic and multidisciplinary approach to reach these industrial targets. Indeed, the AM process involves several design technological issues, like temperature control of the material to be processed, characteristics of the energy source for material transition, control of the power transferred to the material, scanning system's head control, 3D model's layer definition, and generation of the laser point's trajectories. The final product's quality strongly depends on all these aspects, which are synergically linked to each other, as well as on the technical solutions to realize them. The paper presents an interdisciplinary approach to the design of machines for AM, based on the Powder Bed Fusion process and targeted at the industrial field. The technological platforms discussed in the paper are essential for such types of machines. The strategy proposed constitutes a base reference point for the definition of a methodological approach to the design of AM machinery.
\end{abstract}

Keywords: Additive Manufacturing; Selective Laser Sintering/Melting; Driving Technologies; AM Systems Design.

\section{Introduction}

Net Shape Forming (NSF) refers to any manufacturing process, which allows the creation of a product in its finished form without the need for other machining operations. Additive Manufacturing (AM) technology belongs to NSF manufacturing processes' family and it was born in the early 80 s thanks to several inventors who worked on different 3D Printing techniques; by means of AM technology, 3D objects are built by adding layer-upon-layer of material (plastics, metals, ceramics) [1,2].

The family of Additive Manufacturing technologies is quite broad, and it is characterized by several different kinds of processes, according to the specific technology used. As an example, we can talk about selective laser sintering (SLS), selective laser melting (SLM), electron beam melting (EBM), Direct Metal Deposition (DMD), Fused Deposition Modelling (FDM), Stereolithography (SLA), etc. To clearly categorize the AM processes, the ISO/ASTM 52900:2015 standard has defined seven different kinds of processes [3].

The application and implementation of each kind of process leads to several peculiar technological problems that, to get good results in terms of product quality and productivity, must be tackled and solved. In general, they concern:

* Corresponding author: paolo.righettini@unibg.it

\section{doi http://dx.doi.org/10.28991/HIJ-2021-02-01-03}

$>$ This is an open access article under the CC-BY license (https://creativecommons.org/licenses/by/4.0/).

(C) Authors retain all copyrights. 
temperature control of the material to be processed; characteristics of the energy source for material transition; control of the power transferred to the material; scanning system's head control; 3D model's layer definition; and generation of the laser point's trajectories (when a laser is used as an energy source). All these aspects have the same importance in guaranteeing the quality of the product, and they are synergically linked to each other. As an example, the power of the energy source and the temperature of the material are strongly related; the power transferred to the material is related to the trajectories' running speed; the 3D model's layer definition influences the resolution of the positioning system; on the performance and control of the scanning head, the accuracy of the trajectories depends.

The analysis of the influence of these process characteristics on the product has been, and still is, strongly investigated by researchers, along with the way to control the process parameters [4]. Just as an example, investigations concerning heat transfer by means of thermography [5, 6], and analysis of the effect of laser's power on temperature and material defects were made [7]. In addition, in the recent years, researchers have investigated the influence of the process parameters on the dimensional accuracy [8]. Besides the process parameters, some researchers have also investigated the influence of the building orientation on the mechanical characteristics of the product [9]. Moreover, in the last years the effort of researchers seems to be focused on investigations concerning new methods to control the quality of the product in-situ [10-12].

The quality of the product strongly depends on all the aforementioned aspects as well as on the technical solution to realize them. In other words, the machine implementing the specific AM technological process is crucial for the product's manufacturing. From the early stages of machine's concept, a multidisciplinary and synergistic approach which allows to consider all the different aspects involved in the process must be followed. According to such an approach the synergistic application of different disciplines like mechanics, electronics, control, and computer engineering in the development of products and systems through an integrated design approach is needed.

This paper mainly focuses on the AM technology known as Powder Bed Fusion, in which thermal energy selectively fuses regions of a powder bed as stated by ASTM F2792 [3]; more in detail the Selective Laser Melting (SLM) and Selective Laser Sintering (SLS) are addressed. The fundamental aspects that must be considered in the design process of an AM machine are highlighted, and the used design approach is discussed.

\section{General Machine's Configuration}

As explained in Edgar and Tint (2015) study [2], the production process of an AM machine can be summarized as follows: 3D CAD model preparation and STL conversion, slicing of the STL file, machine set-up, building of the product, removing from the machine, possible post-processing operations.

With particular reference to a Selective Laser Melting (SLM) or Selective Laser Sintering (SLS) machine, the product's building consists of heating a portion of the powder bed by means of a laser moving the scanning head according to the "sliced" 3D CAD model, lowering the platform of the building volume, dispensing another layer of powder and recoating with another powder's layer. Then the working cycle restarts.

Figure 1a and $1 \mathrm{~b}$ show a conceptual sketch of a machine. In particular, Figure 1a focuses on the laser source, the scanning head and the layer heated by the laser; Figure $1 \mathrm{~b}$ highlights the building powder container and the relevant lowering platform, the dispensing container and the recoating device.

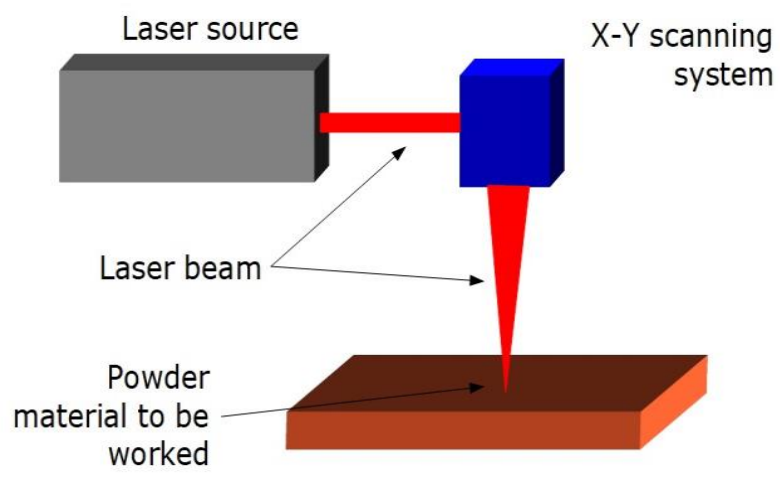

(a)

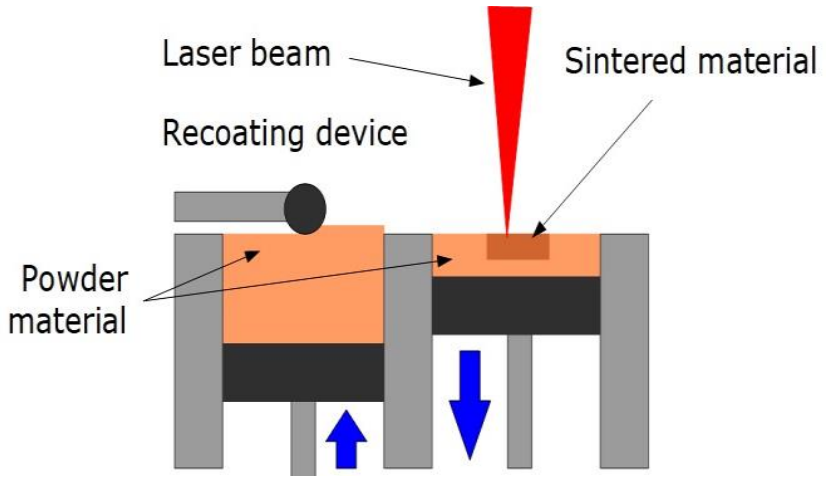

(b)

Figure 1. Conceptual scheme of a machine

From a simply conceptual point of view, a machine for Additive Manufacturing does not seem to be strongly complex; a specific sequence of motions and operations allow to realize the building process. Actually, there are some technological aspects that are very crucial to have a successful production process. 
Again with reference to a Powder Bed Fusion machine, one of those crucial aspects is related to SLS/SLM technology: it is based on the use of a laser source, whose power depends on the powder material processed [13], for sintering portions of a surface of pre-heated powder material. Figure 2 schematically shows the conceptual scheme of sintering process. Hence the thermal energy control along with the laser energy control are very important issues of a machine. Besides the thermal energy control, another important issue concerns the control of the laser beam, which is the control of the laser scanning head and the choice of the path to be sintered. Moreover, the slicing operation of the STL 3D model is very important for the quality of the product, in particular the choice of the slicing step and the algorithm to extract layers from the STL model.

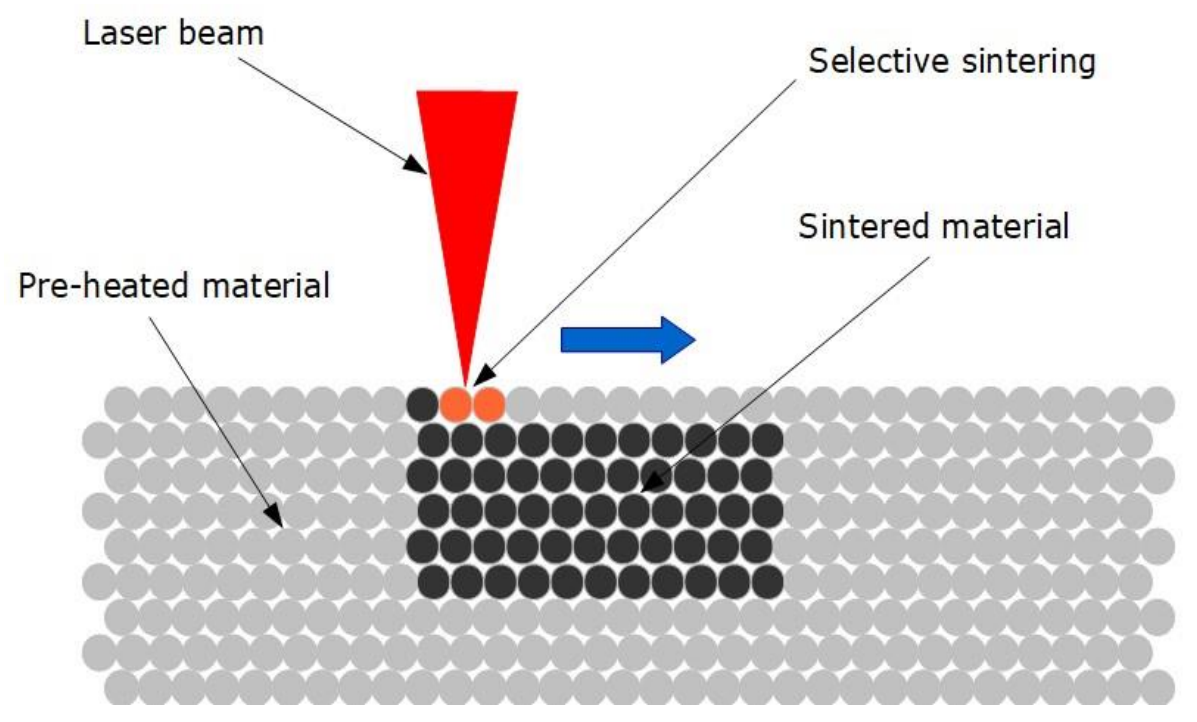

Figure 2. Conceptual scheme of the sintering process

\section{Thermal Energy Control}

A Power Bed Fusion process needs thermal energy to bring the material powder within a specific range of temperature to make sintering or melting occur. As a matter of fact, the melting temperature must be reached for SLM, while the mechanism of sintering is the fusion of powder particles in their solid state at high temperature, between one half of the melting temperature and the melting temperature, which depends on the powder material.

Depending on the powder temperature and on the time at which the temperature is maintained, the sintering process gives different results, mainly concerning the porosity, which is the density of the product. To have products with high density, high sintering temperatures and high sintering times are needed; on the contrary, fusion by melting is quicker. That is why sintering is rarely used as the primary fusion mechanism [2].

However, it is still an important mechanism in most Power Bed Fusion processes, and the design of a machine implementing this technology, must properly take into account some important effects that sintering has on the product building: also the loose powder within the building container, and not only the one that has to be sintered, begins sintering. This means that the dimensional characteristics change leading to a change of the behavior when used as recycled powder for another production process. Another important aspect is related to the growth of a "skin" around the built part; when the desired cross-section is fused, the loose surrounding powder increases its temperature and, mainly when melting is the primary fusion mechanism, it remains at high temperature for long time; it results in part growth. Moreover, since the lower layers (i.e. the first fused) are maintained to high temperature for a time longer than the upper ones, the product part has higher density in lower regions rather than in the upper ones. Powder bed temperature has a great influence also on the geometry of the single fused layer; as a matter of fact, the fused cross section could be subjected to curling effects due to the temperature difference between the loose powder and fused cross section [14].

All these typical aspects highlighted, it is clear that temperature and thermal energy needed to get it are important issues of a PBF process [4]. Hence, from a design point of view, the choice of the thermal source to guarantee the proper temperature of the powder bed is crucial as well as the methods to control its distribution on the powder bed. The issue of temperature monitoring has been dealt with in literature by several authors as [5-7, 10, 12]. Infrared thermal cameras and pyrometers are commonly used. As far as thermal source is concerned, infrared lamps are generally used for polymers, sometimes in conjunction with resistors to prevent border effects around the building volume. 


\section{Laser Energy Control}

In Powder Bed Fusion process in which melting is the primary fusion mechanism the powder bed is maintained at a specific temperature, lower than melting point, and a laser, or an electron beam in case of EBM (Electron Beam Melting), is used to give the powder the energy increment needed for melting. Hence the control of laser energy is a very important issue to guarantee the proper powder melting. Laser energy needed depends on powder characteristics like thermal conductivity, absorptivity and reflectivity as an example a powder with high thermal conductivity requires a laser with higher power because heat dissipates very fast [14]. Moreover, the total amount of energy that is absorbed by the powder depends on laser beam speed, on beam spot size and on scan spacing [2, 14]. Hence, rather than the absolute laser energy or power, the most important parameter is the laser energy density. A simplified model for the energy density on the surface of the powder bed can be written as $[2,14]$ :

$\mathrm{E}_{s}=\frac{\mathrm{W}_{L}}{v_{s} \delta}$

Where $\mathrm{E}_{s}\left(J / m^{2}\right)$ is the surface energy density, $\mathrm{W}_{L}(W)$ is the laser power, $v_{s}(\mathrm{~m} / \mathrm{s})$ is the scan speed and $\delta(m)$ is the spacing between adjacent scan lines.

A similar equation can be written for the energy density across the thickness:

$\mathrm{E}_{T}=\frac{\mathrm{W}_{L}}{v_{S} \delta \sigma}$

Where $\mathrm{E}_{T}\left(\mathrm{~J} / \mathrm{m}^{3}\right)$ is the surface energy density across thickness and $\sigma(m)$ is the layer thickness. The laser effect depends also on the spot size, which must be strictly related to the distance between adjacent scan lines. As a matter of fact, if the spot size is smaller than scan spacing, it results in inappropriate fusing and porosity of the product. A bigger spot size requires more power from the laser to have the same energy density. Moreover, the spot size is related to resolution and to production time; the smaller the spot size, the higher the resolution, but the longer the production time. Hence a proper balance between these different aspects must be sought after and reached. All things considered, the proper choice of laser parameters has a very high influence on the results obtained on the product.

\section{Laser Beam Control}

For a proper implementation of Powder Bed Fusion technology, and in particular Selective Laser Sintering/Melting, besides the control of the laser energy and parameters, also the control of the path followed by the laser beam is very important; it is still a research issue [15]. As a matter of fact, to properly fuse the powder within the desired cross section, the laser beam must move along a path, filling the cross-section itself. According to the kind of path, different scanning strategies can be defined. The main differences concern the kind of path (linear, spiral, etc.), the distance between adjacent lines, possible division of the cross-section area into sub-sections [2, 14]. One of the process characteristics influenced by the chosen scanning strategy is the productivity; as a matter of fact, a longer path leads to a longer time to fill the cross-section area. Besides the length of the path, also its shape is very important; as an example, if the cross section is filled just with linear parallel paths, it results in shrinkage stress and anisotropic strength, leading to warp and distortion. Moreover, linear paths aren't the best choice in case of presence of cavities inside the cross section; as a matter of fact, as it's schematically shown in Figure 3, the laser beam should travel along the empty area with the laser switched off. This results in wasting of time while, frequently switching on and off, the laser reduces its lifetime.

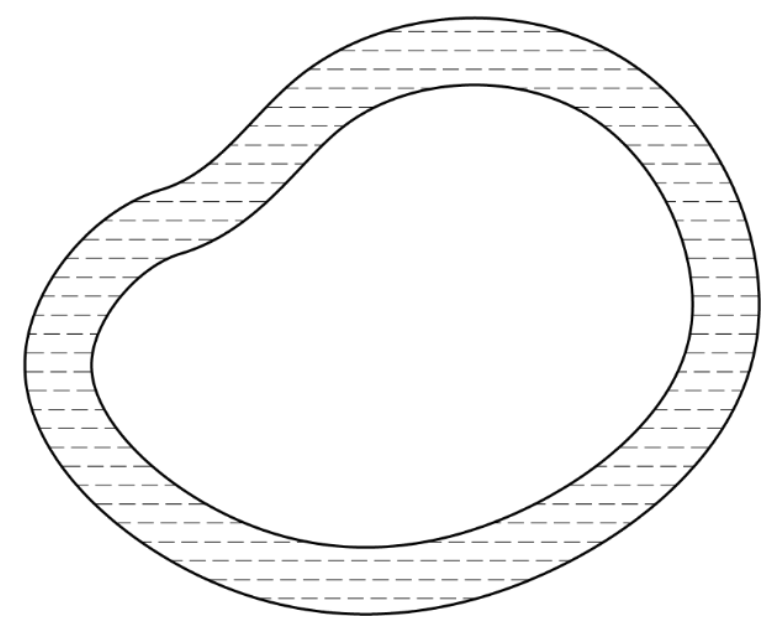

Figure 3. Example of cross section with cavity (linear paths in dotted lines) 
Another parameter already mentioned in the previous section is the distance between adjacent scan lines, which is how dense the path is. The density of the path, being related to the path's length, influences the building time of the product, i.e. the productivity. A less dense path increases the productivity but, depending on the spot size, it could lead to an inappropriate powder fusion and hence to a product with high porosity and inadequate mechanical characteristics. To find a good balance between these different aspects, a scanning strategy could include variable distance between adjacent lines, according to the position inside the area. An example is shown in Figure 4, where paths near to the border have a distance lower than the ones in the inner part of the area. The choice to lower the distance near to the borders allows to have good finishing of the external surface of the product.

However, the results obtained with a specific path could be different depending on its position inside the building volume. As a matter of fact, heat transmission is strongly affected by the position and so the effect on the resulting product. The design process of a machine cannot disregard all these important issues concerning scanning strategies, mostly when a synergistic multidisciplinary approach is followed.

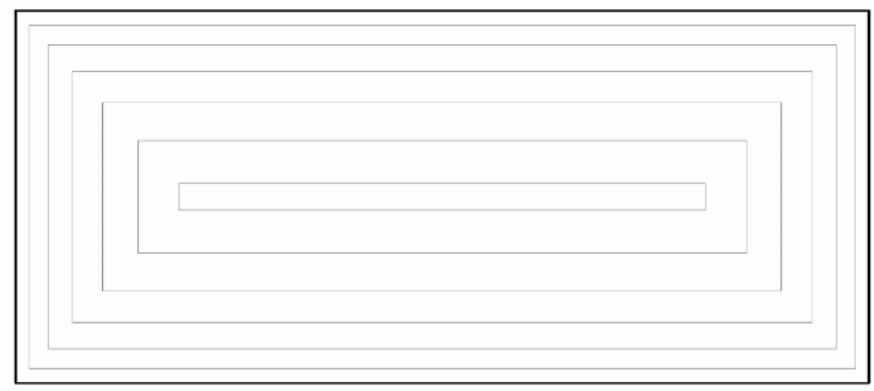

Figure 4. Path with variable distances between lines

\section{3D Model Slicing}

In previous sections, important issues concerning Powder Bed Fusion technology and the process design of the relevant machines have been highlighted. One of these aspects is the layer thickness; as explained in the laser section, it is related to the volume energy density. In general, the choice of the layer thickness is done in a pre-processing phase of the product building, precisely during the STL file manipulation phase. On the STL file, the machine's control software has to perform a slicing operation in order to get the cross-section areas to be sintered (Figure 5). Obviously, the layer thickness influences also the pre-processing time needed.

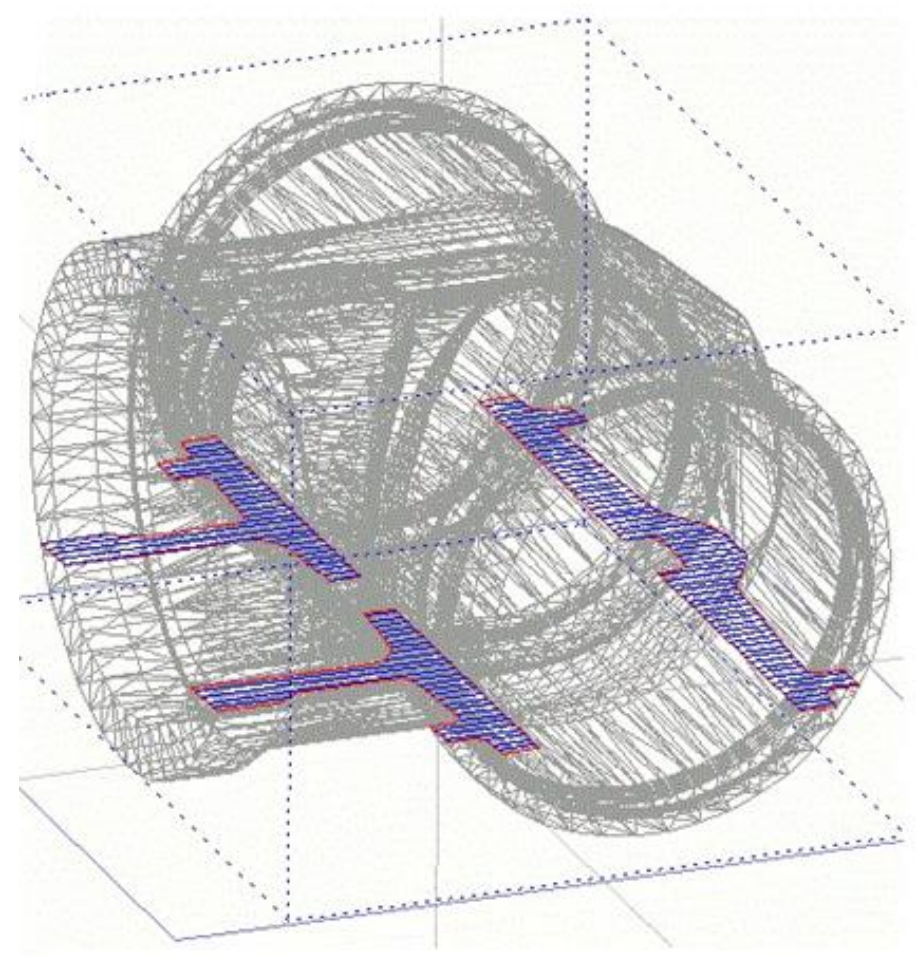

Figure 5. Slicing of a STL file 
As well known, in a STL file, surfaces are approximated by a mesh of triangles. The slicing software must recognize intersection between the cutting plane and the triangles giving as output the shape of the cross section to be sintered.

From the choice of layer thickness, the productivity, and the resolution of the outer surface of the product depend. As a matter of fact, the higher the thickness, the shorter the product building time, but with high layer thickness the well-known staircase effect becomes more evident. Figure 6 schematically shows a vertical plane section where the solid line represents the ideal profile while dotted line is the actual one. The staircase effect is clearly showed: the higher the layer thickness, the larger the gaps between ideal and actual profile.

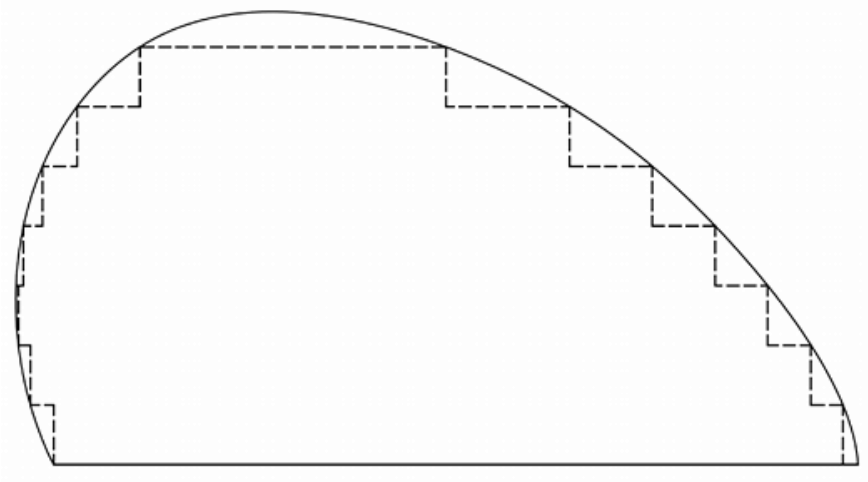

Figure 6. Staircase effect

The choice of the thickness depends on the building strategy used, which is for example the growth direction of the product, which influences the staircase effect. As shown in Figure 7, the same product positioned in a different way can lead to dramatically different results in terms of geometric quality.

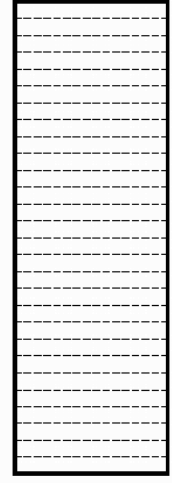

(a)

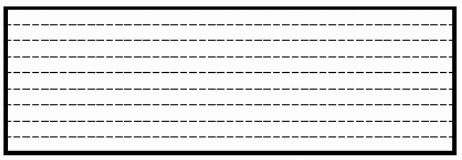

(b)

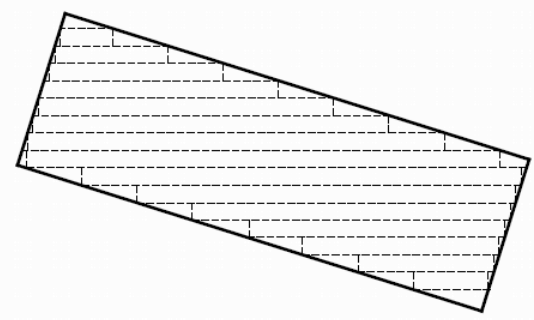

(c)

Figure 7. Positioning into build chamber

In particular, Figure 7c shows, for the same product shape, how much the orientation influences the staircase effect. Moreover, between Figure $7 \mathrm{a}$ and $7 \mathrm{~b}$, it is also clear that the best orientation is (7b), allowing to have a very low number of layers. It should be also noticed that orientation (7a) and (7b), not having problems related to staircase effect, could allow to use layers with higher thickness than orientation (7c). So, the building strategy has a big influence on the production time because, according to the orientation of the product the number of layers could be definitely different.

\section{Machine Industrial Design}

From the technological point of view, the problems related to Additive Manufacturing and, in particular to Powder Bed Fusion, are clear. As seen in the previous sections there are issues concerning thermal control, laser energy and relevant parameters, laser beam control, scanning path definition and so on.

To design and develop a machine that implements PBF technology, it is necessary to set-up proper and efficient technical solutions to transform technological needs in an industrial machine as schematically represented in Figure 8. 


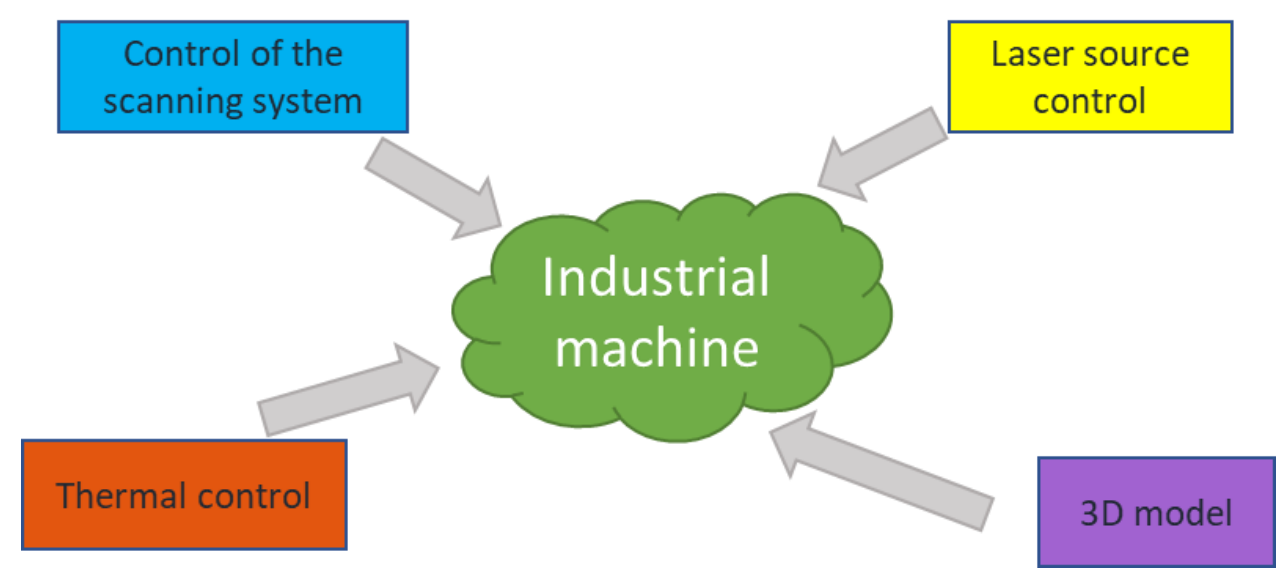

Figure 8. From technological issues to industrialization

In literature, papers concerning the design of industrial machines aren't so widespread, probably for confidentiality reasons, while it is possible to find some works concerning development of prototypes even characterized by new solutions at the aim to improve the production process [16-20], or machines designed and built mainly for research purposes [21], or guidelines for the design of a proper plant layout [22].

All the aspects involved in the design of an Additive Manufacturing machine cannot be dealt with separately, but they are linked each other and not independent. A synergistic approach is needed; even from the concept phase of the machine the technological issues and the relevant technical solutions must be tackled as a whole. One of the main aspects concerns the optimal choice of the motor-transmission system [23].

As an example, concerning the displacement of the building platform, it depends on layer thickness which generally varies between 100 and $300 \mu \mathrm{m}$. Hence a very accurate transmission system is needed; as an example, a recirculating balls screw coupled with a brushless servomotor, which is a system characterized by high stiffness, could be used. Another important motion is the one devoted to powder recoating; in this case, the axis should be as fast as possible to reduce the time of this process step, that isn't a product building step. Also in this case, a solution with high performances should be used; again, a brushless servomotor with a stiff transmission like a recirculating balls screw would be a good solution.

For powder distribution, instead of having a container with a moving piston near the building container, the best solution would be to leave the powder fall down from an hopper in front of the recoating mechanism; a simpler solution without the need for another axis, the one to move the piston. In order to reduce the waste of time, two hoppers could be used, one on the left and one on the right of the building volume; in this way, the recoating axis lays the powder in each direction without having a working stroke and a return stroke.

According to a synergistic multidisciplinary approach [24], simulation can be carried on predicting the behavior of the system. Besides simulations concerning the axis motion and the production cycle of the machine, also thermal simulation should be carried on to define the position and the power of the heating elements like infrared lamps and resistors that guarantee the proper temperature distribution on the powder bed.

Then the control software must manage all the functionalities required; it has to perform the pre-processing activities like STL slicing, to allow parameters setting, temperature control, laser control, scanning path control, axes motion control and it has to coordinate the different steps of the production process. Hence, since the early stage of the design process, the concept of the software must take into consideration all the functionalities that must be guaranteed to the system.

\section{Conclusion}

In this paper, the main technological issues related to Powder Bed Fusion process have been highlighted. With particular reference to a Selective Laser Melting (SLM) or Selective Laser Sintering (SLS) machine, such issues include thermal energy control, laser energy control, laser beam control, 3D model slicing, and building orientation of the product in the building chamber. A potential designer of such machines must tackle all these issues. None is the most important: each technological issue is equally important with the aim of guaranteeing the quality of the product. They are synergistically linked each other, hence a synergistic multidisciplinary approach must be used to properly manage the technologies and design the machine.

In the literature, papers concerning the analysis of single technological problems are widespread, while there is a lack of papers dealing with a comprehensive view of all the technological aspects involved in the proper design of an AM machine. A comprehensive synergistic multidisciplinary approach has a fundamental role in the design of such 
machines; since the early stage of concept design, all technological aspects that synergistically interact with each other must be taken into account as a whole, along with the relevant technical solution. That is the way to make an optimal design and to develop an efficient machine with high performance. The present paper has exactly this objective, to give an overall view of the design process of an AM machine, highlighting all the problems related to the realization of an industrial machine. As a matter of fact, the design of an industrial machine for Additive Manufacturing, and in particular Powder Bed Fusion, needs to transform the technological issues into technical solutions which allow their implementation with reference not to a prototypical or research environment, but to an industrial one, that is, moving the application from laboratories to the plant floor.

\section{Acknowledgements}

The authors are grateful to the University of Bergamo and to its Department of Engineering and Applied Sciences for the annual research funding that has made possible the realization of this work.

\section{Declaration of Competing Interest}

The authors declare that they have no known competing financial interests or personal relationships that could have appeared to influence the work reported in this paper.

\section{References}

[1] Molitch-Hou, M. (2018). Overview of additive manufacturing process. Additive Manufacturing, 1-38. doi:10.1016/b978-0-12812155-9.00001-3.

[2] Edgar, J., \& Tint, S. (2015). Additive Manufacturing Technologies: 3D Printing, Rapid Prototyping, and Direct Digital Manufacturing, 2nd Edition. Johnson Matthey Technology Review, 59(3), 193-198. doi:10.1595/205651315x688406.

[3] Standard ISO/ASTM 52900:2015 (ASTM F2792). (2015). Additive Manufacturing—General Principles—Terminology. ISO: Geneva, Switzerland.

[4] Zhang, J., Song, B., Wei, Q., Bourell, D., \& Shi, Y. (2019). A review of selective laser melting of aluminium alloys: Processing, microstructure, property and developing trends. Journal of Materials Science \& Technology, 35(2), $270-284$. doi:10.1016/j.jmst.2018.09.004.

[5] Krauss, H., Eschey, C., Zaeh, M. F. (2012). Thermography for Monitoring the Selective Laser Melting Process, Proceedings of the 23rd Annual International Solid Freeform Fabrication (SFF) Symposium - An Additive Manufacturing Conference, Austin, Texas, USA, August 6-8.

[6] Zeng, K., Pal, D., Stucker, B. (2012). A review of thermal analysis methods in Laser Sintering and Selective Laser Melting, Proceedings of the 23rd Annual International Solid Freeform Fabrication (SFF) Symposium - An Additive Manufacturing Conference, Austin, Texas, USA, August 6-8.

[7] Islam, M., Purtonen, T., Piili, H., Salminen, A., \& Nyrhilä, O. (2013). Temperature Profile and Imaging Analysis of Laser Additive Manufacturing of Stainless Steel. Physics Procedia, 41, 835-842. doi:10.1016/j.phpro.2013.03.156.

[8] Sharma, V., \& Singh, S. (2020). To Study the Effect of SLS Parameters for Dimensional Accuracy. Advances in Materials Processing, 165-173. doi:10.1007/978-981-15-4748-5_17.

[9] Morettini, G., Javad Razavi, S. M., \& Zucca, G. (2019). Effects of build orientation on fatigue behavior of Ti-6Al-4V as-built specimens produced by direct metal laser sintering. Procedia Structural Integrity, 24, 349-359. doi:10.1016/j.prostr.2020.02.032.

[10] Everton, S. K., Hirsch, M., Stravroulakis, P., Leach, R. K., \& Clare, A. T. (2016). Review of in-situ process monitoring and insitu metrology for metal additive manufacturing. Materials \& Design, 95, 431-445. doi:10.1016/j.matdes.2016.01.099.

[11] Repossini, G., Laguzza, V., Grasso, M., \& Colosimo, B. M. (2017). On the use of spatter signature for in-situ monitoring of Laser Powder Bed Fusion. Additive Manufacturing, 16, 35-48. doi:10.1016/j.addma.2017.05.004.

[12] Grasso, M., Demir, A. G., Previtali, B., \& Colosimo, B. M. (2018). In situ monitoring of selective laser melting of zinc powder via infrared imaging of the process plume. Robotics and Computer-Integrated Manufacturing, 49, $229-239$. doi:10.1016/j.rcim.2017.07.001.

[13] Pinkerton, A. J. (2016). Lasers in additive manufacturing. Optics \& Laser Technology, 78, 25-32. doi:10.1016/j.optlastec. 2015.09.025.

[14] Padmakumar, M. (2020). Additive manufacturing of tungsten carbide hardmetal parts by selective laser melting (SLM), selective laser sintering (SLS) and binder jet 3D printing (BJ3DP) techniques. Lasers Manuf. Mater. Process, 7, 338-371. doi:10.1007/s40516-020-00124-0. 
[15] Ezair, B., Fuhrmann, S., \& Elber, G. (2018). Volumetric covering print-paths for additive manufacturing of 3D models. Computer-Aided Design, 100, 1-13. doi:10.1016/j.cad.2018.02.006.

[16] Fish, S., Booth, J. C., Kubiak, S. T., Wroe, W. W., Bryant, A. D., Moser, D. R., \& Beaman, J. J. (2015). Design and subsystem development of a high temperature selective laser sintering machine for enhanced process monitoring and control. Additive Manufacturing, 5, 60-67. doi:10.1016/j.addma.2014.12.005.

[17] Wulle, F., Coupek, D., Schäffner, F., Verl, A., Oberhofer, F., \& Maier, T. (2017). Workpiece and Machine Design in Additive Manufacturing for Multi-Axis Fused Deposition Modelling. Procedia CIRP, 60, 229-234. doi:10.1016/j.procir.2017.01.046.

[18] Yamazaki, T. (2016). Development of A Hybrid Multi-tasking Machine Tool: Integration of Additive Manufacturing Technology with CNC Machining. Procedia CIRP, 42, 81-86. doi:10.1016/j.procir.2016.02.193.

[19] Mazumder, J. (2015). Design for Metallic Additive Manufacturing Machine with Capability for "Certify as You Build." Procedia CIRP, 36, 187-192. doi:10.1016/j.procir.2015.01.009.

[20] Phillips, T., Fish, S., \& Beaman, J. (2018). Development of an automated laser control system for improving temperature uniformity and controlling component strength in selective laser sintering. Additive Manufacturing, 24, 316-322. doi:10.1016/j.addma.2018.10.016.

[21] Hussain, G., Khan, W. A., Ashraf, H. A., Ahmad, H., Ahmed, H., Imran, A., .. Abbas, G. (2019). Design and development of a lightweight SLS 3D printer with a controlled heating mechanism: Part A. International Journal of Lightweight Materials and Manufacture, 2(4), 373-378. doi:10.1016/j.ijlmm.2019.01.005.

[22] Kowalski, A., \& Waszkowski, R. (2020). Layout Guidelines for 3D Printing Devices. Applied Sciences, 10(18), 6333. doi:10.3390/app10186333.

[23] Roos, F., Johansson, H., \& Wikander, J. (2006). Optimal selection of motor and gearhead in mechatronic applications. Mechatronics, 16(1), 63-72. doi:10.1016/j.mechatronics.2005.08.001.

[24] De Silva, C. W. (2005). Mechatronics: an integrated approach, CRC Press - Taylor \& Francis Group, Florida, United States. 